\begin{tabular}{|c|l|}
\hline Title & A coustic phonons in nanowire superlattices: A zimuthally symmetric torsional modes \\
\hline Author(s) & Mizuno, Seiji \\
\hline Citation & $\begin{array}{l}\text { Physical Review B, 71,085303 } \\
\text { https://doi.org/10.1103/PhysRevB.71.085303 }\end{array}$ \\
\hline Issue Date & 2005-02-04 \\
\hline Doc URL & http://hdl.handle.net/2115/5707 \\
\hline Rights & Copyright $\odot 2005$ A merican Physical Society \\
\hline Type & article \\
\hline File Information & PRB71.pdf \\
\hline
\end{tabular}

Instructions for use 


\title{
Acoustic phonons in nanowire superlattices: Azimuthally symmetric torsional modes
}

\author{
Seiji Mizuno \\ Department of Applied Physics, Graduate School of Engineering, Hokkaido University, Sapporo 060-8628, Japan
}

(Received 7 June 2004; revised manuscript received 4 October 2004; published 4 February 2005)

\begin{abstract}
We study theoretically vibrational modes in a nanowire superlattice consisting of an alternate stacking of two cylindrical layers. We focus on azimuthally symmetric torsional modes and calculate the phonon dispersion relations analytically. We also derive simple expressions for the frequency gaps generated in the nanowire superlattice. Moreover, we calculate the transmittance of phonons propagating through a nanowire superlattice with the finite number of periods. Based on our calculated results, effects of the superlattice longitudinal confinement and the radial confinement are examined.
\end{abstract}

DOI: 10.1103/PhysRevB.71.085303

PACS number(s): 68.65.Cd, 63.22. $+\mathrm{m}, 62.65 .+\mathrm{k}, 63.20 .-\mathrm{e}$

\section{INTRODUCTION}

Recently, realization of one-dimensional compositionally modulated heterostructures is generating a great deal of interest for potential nanoelectronics and photonics. For example, growth of $\mathrm{GaAs} / \mathrm{GaP},{ }^{1} \mathrm{Si} / \mathrm{SiGe},{ }^{2} \mathrm{InAs} / \mathrm{InP},{ }^{3}$ and $\mathrm{ZnSe} /$ CdSe (Ref. 4) nanowire superlattice (NWSL) structures have been reported. A theory of electrons in NWSLs has been presented and these structures are shown to be radically different from plain nanowires and quantum well structures in their electronic, optical, and transport properties. ${ }^{5}$

Furthermore, we can expect that this structure yields interesting physical effects on the phonon properties. It is also practically important to understand the transmission properties of phonons in NWSLs. For example, phonons play important roles in micro/nano electromechanical systems (MEMS/NEMS), which have attracted much attention in recent years. ${ }^{6}$ The characteristics of such nano electromechanical devises are determined by the thermal effects. By utilizing the superlattice structure embedded in the nanowire, it is possible to control the behavior of phonons in the nano electromechanical devises. In the periodic superlattice structure, Bragg reflections occur for long wavelength phonons. In other words, the periodic superlattice structure acts as an opaque barrier for phonons within the frequency gaps induced by the periodicity much longer than the lattice spacing. This suggests the potential for designing various phonon optics devices for coherent phonon generation and control, such as phonon filters, mirrors, and resonators. ${ }^{7}$

The aim of the present paper is to study the acoustic phonons in NWSLs theoretically. In particular, we focus on azimuthally symmetric torsional modes. These modes can be excited with the use of a resonator generating torsional vibrations of a suspended wires, which was developed by Cleland et al. ${ }^{6}$ As for a "plain" cylindrical isotropic wire with stress-free boundaries, vibrational properties have been already studied. ${ }^{8,9}$ For the azimuthally symmetric torsional modes in the plain wire, it has been shown that displacement and stress fields and also phonon dispersion relation can be analytically obtained. In the present paper, we study analytically these vibrational modes generated in the NWSLs, and examine effects of both the superlattice longitudinal confinement and the radial confinement.

The outline of this paper is as follows: In Sec. II A, we briefly summarize the vibrational modes in a plain wire. In
Sec. II B, we develop the mathematical analysis of the phonon dispersion relations of NWSLs and the transmittance of NWSLs with the finite number of periods. Approximate expressions for the gap frequencies are also derived. In Sec. III, the numerical examples based on our formula are presented, and the vibrational properties are discussed. In Sec. IV, a summary and conclusions are given.

\section{FORMULATION}

\section{A. Free isotropic cylinder}

For the later use, we summarize in this section results for vibrational modes in a cylindrical isotropic wire with stressfree boundaries. For isotropic materials, it is convenient to apply the potential theory method., ${ }^{9,10}$ The displacement fields $\boldsymbol{u}$ can be expressed in terms of potential functions:

$$
\boldsymbol{u}=\nabla \phi_{1}+\nabla \times\left(\phi_{2} \hat{z}\right)+\nabla \times \nabla \times\left(\phi_{3} \hat{z}\right),
$$

where $\hat{z}$ is the unit vector in the $z$ direction. Each potential function $\phi_{i}(i=1,2,3)$ satisfies the following wave equation:

$$
\left(\nabla^{2}+\frac{\omega^{2}}{v_{i}^{2}}\right) \phi_{i}=0
$$

where $v_{1}=v_{l}, v_{2}=v_{3}=v_{t}$ are the sound velocities for the longitudinal and transverse modes, respectively. The appropriate solutions of Eq. (2) are well known, that is,

$$
\phi_{i}=C_{i} J_{p}\left(k_{i} r\right) e^{i(p \theta+q z)},
$$

in cylindrical coordinate. Here $C_{i}$ is a constant, $J_{p}\left(k_{i} r\right)$ is the Bessel function of the first kind, $p$ is an integer representing the $p$-fold rotational symmetry, $q$ is the wave number in the $z$ direction, and

$$
\begin{gathered}
k_{1}=\sqrt{\frac{\omega^{2}}{v_{\ell}^{2}}-q^{2}} \equiv k_{\ell}, \\
k_{2}=k_{3}=\sqrt{\frac{\omega^{2}}{v_{t}^{2}}-q^{2}} \equiv k_{t}
\end{gathered}
$$

mean the wave numbers of the longitudinal and transverse modes in the radial direction, respectively. Substitution of 
Eq. (3) into Eq. (1) gives the phonon displacement field $\boldsymbol{u}=\left(u_{r}, u_{\theta}, u_{z}\right)$ in the cylindrical coordinate:

$$
\begin{gathered}
u_{r}=\left(C_{1} \frac{\partial J_{p}\left(k_{\ell} r\right)}{\partial r}+C_{2} \frac{i p}{r} J_{p}\left(k_{t} r\right)+C_{3} i q \frac{\partial J_{p}\left(k_{t} r\right)}{\partial r}\right) \times e^{i(p \theta+q z)}, \\
u_{\theta}=\left(C_{1} \frac{i p}{r} J_{p}\left(k_{\ell} r\right)-C_{2} \frac{\partial J_{p}\left(k_{t} r\right)}{\partial r}-C_{3} \frac{p q}{r} J_{p}\left(k_{t} r\right)\right) \times e^{i(p \theta+q z)}, \\
u_{z}=\left(C_{1} i q J_{p}\left(k_{\ell} r\right)+C_{3} k_{t}^{2} J_{p}\left(k_{t} r\right)\right) \times e^{i(p \theta+q z)} .
\end{gathered}
$$

For $p=0$ (i.e., azimuthally symmetric modes), $C_{2}$ is decoupled from $C_{1}$ and $C_{3}$, and the lattice displacement field $u_{\theta}$ is expressed as

$$
u_{\theta}=-C_{2} \frac{\partial J_{0}\left(k_{t} r\right)}{\partial r} e^{i q z}=C_{2} k_{t} J_{1}\left(k_{t} r\right) e^{i q z}
$$

The corresponding stress field $\sigma_{r \theta}$ is

$$
\sigma_{r \theta}=-C_{2} \mu k_{t}^{2} J_{2}\left(k_{t} r\right) e^{i q z},
$$

where $\mu=\rho v_{t}^{2}$ and $\rho$ is the mass density. In the present study, we consider these modes, namely, azimuthally symmetric torsional modes. There are mainly two reasons for considering these modes. First, we can with proceed the analytical calculation also for the NWSLs. Second, a resonator generating the torsional vibrations of a suspended wire was actually developed. ${ }^{6}$

For a wire with free boundaries, the components of the stress should vanish at the surface of the cylinder $r=R$. This boundary condition leads to

$$
k_{t}=\frac{f_{2, s}}{R},
$$

where $f_{2, s}$ is the $s$ th root of the equation $J_{2}=0$, e.g., $f_{2,0}=0$, $f_{2,1}=5.136, f_{2,2}=8.418$, etc. From Eqs. (5) and (11), the dispersion relation is analytically obtained as

$$
\omega=v_{t} \sqrt{q^{2}+\left(\frac{f_{2, s}}{R}\right)^{2}} .
$$

For $s=0$, Eq. (12) becomes

$$
\omega=v_{t} q
$$

but the corresponding $u_{\theta}$ vanish. However, it is known that the solution

$$
u_{\theta}=C_{2} r e^{i q z}
$$

corresponds to $s=0^{9}$. This can be directly confirmed with the substitution of Eq. (14) into the wave equation. From Eq. (12), it is found that the lowest frequency for the sth mode is given by

$$
\omega_{\min }=v_{t} \frac{f_{2, s}}{R},
$$

because the wave number $q$ should be a real number for the wire with the infinity length. (a)

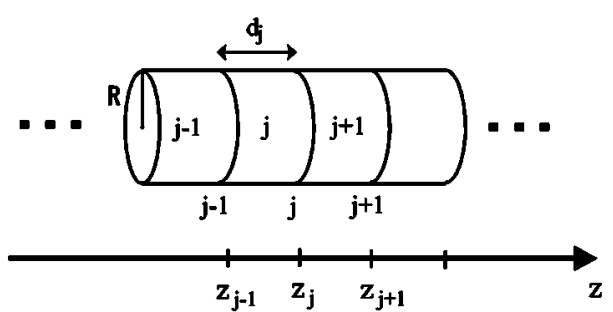

(b)

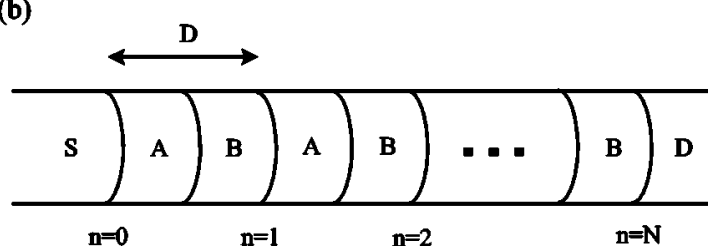

FIG. 1. Nanowire superlattice.

\section{B. Nanowire superlattice}

\section{Dispersion relation}

In this section, we consider the azimuthally symmetric torsional modes in a cylindrical NWSL. The NWSL is modeled as an ideal cylinder with sharp modulation in the longitudinal direction [Fig. 1(a)]. In this case, the lattice displacement $u_{\theta, j}$ and stress $\sigma_{\theta z, j}$ defined in the $j$ th layer of the NWSL are expressed in terms of linear combinations of the transmitted and reflected waves propagating along the $z$ axis:

$$
\begin{gathered}
u_{\theta, j}=F(r)\left(a_{j} e^{i q_{j} z}+b_{j} e^{-i q_{j} z}\right), \\
\sigma_{\theta z, j}=i \mu_{j} q_{j} F(r)\left(a_{j} e^{i q_{j} z}-b_{j} e^{-i q_{j} z}\right),
\end{gathered}
$$

where

$$
F(r)=\left\{\begin{array}{ll}
\frac{f_{2, s}}{R} J_{1}\left(\frac{f_{2, s}}{R} r\right) & \text { for } \quad s \neq 0 \\
r & \text { for } \quad s=0
\end{array} .\right.
$$

Here, $a_{j}$ and $b_{j}$ are the amplitudes of the transmitted and reflected waves, respectively. For given frequencies the wave number $q_{j}$ in the $j$ th layer is determined by the dispersion relation (12):

$$
q_{j}=\sqrt{\left(\frac{\omega}{v_{j}}\right)^{2}-\left(\frac{f_{2, s}}{R}\right)^{2}},
$$

where $v_{j}$ is the sound velocity for the transverse mode. In the present system, $q_{j}$ is allowed to be an imaginary number because the $j$ th layer has boundaries in the $z$ direction.

The lattice displacement $u_{\theta, j}$ and stress $\sigma_{\theta z, j}$ should be continuous at the interfaces of adjacent layers. This boundary condition can be explicitly expressed as

$$
\mathbf{U}_{j}\left(z_{j}\right)=M_{j} \mathbf{U}_{j-1}\left(z_{j-1}\right),
$$

where

$$
\mathbf{U}_{j}(z)=\left(\begin{array}{l}
u_{\theta, j}(z) \\
\sigma_{\theta z, j}(z)
\end{array}\right)
$$

and 


$$
M_{j}=\left(\begin{array}{cc}
\cos \left(q_{j} d_{j}\right) & \frac{1}{\mu_{j} q_{j}} \sin \left(q_{j} d_{j}\right) \\
-\mu_{j} q_{j} \sin \left(q_{j} d_{j}\right) & \cos \left(q_{j} d_{j}\right)
\end{array}\right)
$$

is the transfer matrix of the $j$ th layer.

Using the transfer matrix, we can derive the phonon dispersion relation of a NWSL consisting of an alternate stacking of two cylindrical layers. If the interfaces between unit periods are labeled with $n$ as shown in Fig. 1(b), the relation between $\mathbf{U}_{n}$ and $\mathbf{U}_{n-1}$ can be written as

$$
\mathbf{U}_{n}(n D)=M_{B A} \mathbf{U}_{n-1}[(n-1) D] .
$$

Here, $D\left(=d_{A}+d_{B}\right)$ denotes the thickness of the unit period, where $d_{A}$ and $d_{B}$ are the thicknesses of layers $A$ and $B$, respectively, and $M_{B A}$ is the transfer matrix for the unit period of the NWSL,

$$
M_{B A} \equiv M_{B} M_{A}=\left(\begin{array}{cc}
m_{11} & \frac{1}{\mu_{A} q_{A}} m_{12} \\
\mu_{A} q_{A} m_{21} & m_{22}
\end{array}\right),
$$

where

$$
\begin{aligned}
& m_{11}=\cos \alpha \cos \beta-\frac{\mu_{A} q_{A}}{\mu_{B} q_{B}} \sin \alpha \sin \beta, \\
& m_{12}=\sin \alpha \cos \beta+\frac{\mu_{A} q_{A}}{\mu_{B} q_{B}} \cos \alpha \sin \beta, \\
& m_{21}=-\sin \alpha \cos \beta-\frac{\mu_{B} q_{B}}{\mu_{A} q_{A}} \cos \alpha \sin \beta, \\
& m_{22}=\cos \alpha \cos \beta-\frac{\mu_{B} q_{B}}{\mu_{A} q_{A}} \sin \alpha \sin \beta,
\end{aligned}
$$

and

$$
\begin{aligned}
& \alpha=d_{A} \sqrt{\left(\frac{\omega}{v_{A}}\right)^{2}-\left(\frac{f_{2, s}}{R}\right)^{2}}, \\
& \beta=d_{B} \sqrt{\left(\frac{\omega}{v_{B}}\right)^{2}-\left(\frac{f_{2, s}}{R}\right)^{2}} .
\end{aligned}
$$

In the present system with discrete translational symmetry, the lattice displacement $u_{\theta, n}$ and stress $\sigma_{\theta z, n}$ have to satisfy Bloch's theorem,

$$
\mathbf{U}_{n}=e^{i K D} \mathbf{U}_{n-1},
$$

where $K$ is the Bloch wave number due to the superlattice modulation, which is determined by the periodic boundary condition, $\mathbf{U}_{n+N}=\mathbf{U}_{n}$. On the other hand, the relation between $\mathbf{U}_{n}$ and $\mathbf{U}_{n-1}$ was already given in Eq. (23). Combining Eqs. (23) and (31), we have

$$
\operatorname{det}\left(M_{B A}-e^{i K D}\right)=0 .
$$

Solving this equation, we get the phonon dispersion relation,

$$
\cos K D=\frac{m_{11}+m_{22}}{2}=\cos (\alpha+\beta)-\frac{1}{2} \varepsilon^{2} \sin \alpha \sin \beta,
$$

where

$$
\varepsilon=\sqrt{\frac{\mu_{B} q_{B}}{\mu_{A} q_{A}}}-\sqrt{\frac{\mu_{A} q_{A}}{\mu_{B} q_{B}}} .
$$

Equation (33) can be satisfied for a real $K$ if and only if the right-hand side is less than unity in magnitude. Thus, there will be allowed and forbidden regions of frequencies, i.e., frequency bands and gaps, respectively. In other words, the frequency gaps are defined as the range of frequencies satisfying

$$
\left|\frac{m_{11}+m_{22}}{2}\right|>1
$$

When $s=0, \varepsilon$ can be written as

$$
\varepsilon=\sqrt{\frac{Z_{A}}{Z_{B}}}-\sqrt{\frac{Z_{B}}{Z_{A}}} \equiv \varepsilon_{0}
$$

with the acoustic impedance $Z_{A}=\rho_{A} v_{A}$ and $Z_{B}=\rho_{B} v_{B}$. In other words, $\varepsilon$ means the acoustic mismatch between the constituent layers of the NWSL.

\section{Approximate expression for the gap frequency}

Here, we derive approximate expressions for the gap frequencies. These are useful in understanding the coupling of the longitudinal confinement to radial confinement.

For $s=0$, Eq. (33) becomes the same form as the expression for the dispersion relation of the phonons normally propagating through the one-dimensional SL. ${ }^{11}$ The second term on the right-hand side of Eq. (33) can be regarded as a small perturbation because the acoustic mismatch is small for most of the SLs, that is,

$$
\varepsilon_{0} \ll 1 .
$$

Thus, the frequency at the center of the $m$ th frequency gap is given by

$$
\omega_{m}=m \omega_{1}=m \pi\left(\frac{d_{A}}{v_{A}}+\frac{d_{B}}{v_{B}}\right)^{-1} .
$$

This frequency is known as the Bragg frequency.

Even if $s \neq 0$, the second term on the right-hand side of Eq. (33) is still expected to be a small perturbation though $\varepsilon$ depends on the frequency. The frequency at the center of the $m$ th frequency gap is approximately determined by

$$
\alpha+\beta=m \pi .
$$

With the use of Eqs. (29) and (30), this equation becomes

$$
\frac{d_{A} \omega}{v_{A}} \sqrt{1-\left(\frac{f_{2, s} v_{A}}{R \omega}\right)^{2}}+\frac{d_{B} \omega}{v_{B}} \sqrt{1-\left(\frac{f_{2, s} v_{B}}{R \omega}\right)^{2}}=m \pi .
$$

Equation (40) can be approximated as 


$$
\pi \frac{\omega}{\omega_{1}}-\frac{f_{2, s}^{2}}{2 R^{2} \omega}\left(v_{A} d_{A}+v_{B} d_{B}\right)=m \pi
$$

if

$$
f_{2, s} v \ll R \omega
$$

is satisfied $\left(v=v_{A} \sim v_{B}\right)$. Solving Eq. (41) within the present approximation, we obtain the expression for the Bragg frequency of the NWSL,

$$
\tilde{\omega}_{m}=\omega_{m}+\frac{f_{2, s}^{2}}{2 m \pi R^{2}}\left(v_{A} d_{A}+v_{B} d_{B}\right)
$$

It is found that the second term in Eq. (43) gives the correction due to the radial confinement of phonons. The condition (42) for the validity of Eq. (43) can be rewritten as

$$
\left(\frac{f_{2, s} D}{m R}\right)^{2} \ll 1
$$

because typical frequencies we are interested in are around the Bragg frequency, that is,

$$
\omega \sim \tilde{\omega}_{m} \sim m \omega_{1} \sim m \pi v / D .
$$

In other words, the analytical expression (43) is valid when the effect of the radial confinement is regarded as a small perturbation.

Next, let us estimate the width of the $m$ th frequency gap. Expanding Eq. (33) around $\widetilde{\omega}_{m}$ and neglecting small contribution terms, we have

$$
\frac{m_{11}+m_{22}}{2}=(-1)^{m}\left[1+\frac{\varepsilon_{0}^{2}}{2} \sin ^{2}\left(\omega_{m} d_{A} / v_{A}\right)-\frac{1}{2}\left(\omega-\widetilde{\omega}_{m}\right)^{2} \frac{\pi^{2}}{\omega_{1}^{2}}\right] \text {. }
$$

The derivation of Eq. (46) is given in the Appendix. From this equation, it is shown that Eq. (35) is satisfied for the frequency window,

$$
\widetilde{\omega}_{m}-\Delta_{m} \leqslant \omega \leqslant \tilde{\omega}_{m}+\Delta_{m},
$$

where

$$
\Delta_{m}=\frac{\omega_{1}}{\pi}\left|\varepsilon_{0} \sin \left(\omega_{m} d_{A} / v_{A}\right)\right|
$$

That is, the frequency window defined by Eq. (47) gives the $m$ th frequency gap, or Eq. (48) represents half of the width of the gap.

\section{Transmittance and reflectance}

Next, we consider the case of a periodic NWSL sandwiched between $S$ and $D$ [see Fig. 1(b)], that is, the NWSL has a finite number of period. For this system, the phonon properties are determined in terms of the transfer matrix for the NWSL with $N$ period,

$$
M_{S L} \equiv\left(M_{B A}\right)^{N}=\left(\begin{array}{cc}
M_{11} & \frac{1}{\mu_{A} q_{A}} M_{12} \\
\mu_{A} q_{A} M_{21} & M_{22}
\end{array}\right) .
$$

The matrix elements of $M_{S L}$ can be calculated analytically as

$$
M_{11}=\frac{m_{11}-m_{22}}{2} S(N)+C(N)
$$

$$
\begin{gathered}
M_{12}=m_{12} S(N), \\
M_{21}=m_{21} S(N), \\
M_{22}=-\frac{m_{11}-m_{22}}{2} S(N)+C(N),
\end{gathered}
$$

where

$$
S(N)= \begin{cases}\frac{\sin N P}{\sin P}, & \frac{\left|m_{11}+m_{22}\right|}{2} \leqslant 1 \\ \frac{\sinh N P}{\sinh P}, & \frac{m_{11}+m_{22}}{2}>1 \\ (-1)^{N+1} \frac{\sinh N P}{\sinh P}, & \frac{m_{11}+m_{22}}{2}<-1,\end{cases}
$$

and

$$
C(N)=\left\{\begin{array}{ll}
\cos N P, & \frac{\left|m_{11}+m_{22}\right|}{2} \leqslant 1 \\
\cosh N P, & \frac{m_{11}+m_{22}}{2}>1 . \\
(-1)^{N} \cosh N P, & \frac{m_{11}+m_{22}}{2}<-1
\end{array} .\right.
$$

Here, $P$ is defined by

$$
\begin{array}{ll}
\cos P \equiv \frac{m_{11}+m_{22}}{2}, & \frac{\left|m_{11}+m_{22}\right|}{2} \leqslant 1 \\
\cosh P \equiv \frac{\left|m_{11}+m_{22}\right|}{2}, & \frac{\left|m_{11}+m_{22}\right|}{2}>1 .
\end{array}
$$

The frequencies satisfying the condition $\left|m_{11}+m_{22}\right| / 2$ $>1\left(\left|m_{11}+m_{22}\right| / 2 \leqslant 1\right)$ are inside the frequency gaps (bands), as shown in Eq. (35). Within the frequency bands, $P / D$ is equivalent to the Bloch wave number $K$ [see Eq. (33)]. Within the frequency gaps, on the other hand, $P / D$ represents the decay factor, or the imaginary part of the wave number.

With the use of the transfer matrix, the relation between $\mathbf{U}_{S}(0)$ in the substrate and $\mathbf{U}_{D}(N D)$ in the detector layer is expressed as

$$
\mathbf{U}_{D}(N D)=M_{S L} \mathbf{U}_{S}(0) \text {. }
$$

Here, $\mathbf{U}_{S}$ and $\mathbf{U}_{D}$ are expressed in terms of the transmission amplitude $t(\omega)$ and reflection amplitude $r(\omega)$, respectively,

$$
\begin{gathered}
\mathbf{U}_{S}(z)=\left(\begin{array}{c}
e^{i q_{S} z}+r(\omega) e^{-i q_{S} z} \\
i \mu_{S} q_{S}\left(e^{i q_{S} z}-r(\omega) e^{-i q_{S} z}\right)
\end{array}\right) F(r), \\
\mathbf{U}_{D}(z)=\left(\begin{array}{c}
t(\omega) e^{i q_{D} z} \\
i \mu_{D} q_{D} t(\omega) e^{i q_{D} z}
\end{array}\right) F(r) .
\end{gathered}
$$

Combining Eqs. (57)-(59), we can obtain the expressions for $t(\omega)$ and $r(\omega)$ as 


$$
\begin{aligned}
& t(\omega)=\frac{2 i e^{-i k_{D} L}}{\left(\frac{\mu_{D} q_{D}}{\mu_{A} q_{A}} M_{12}-\frac{\mu_{A} q_{A}}{\mu_{S} q_{S}} M_{21}\right)+i\left(M_{22}+\frac{\mu_{D} q_{D}}{\mu_{S} q_{S}} M_{11}\right)}, \\
& r(\omega)=\frac{\left(\frac{\mu_{D} q_{D}}{\mu_{A} q_{A}} M_{12}+\frac{\mu_{A} q_{A}}{\mu_{S} q_{S}} M_{21}\right)+i\left(M_{22}-\frac{\mu_{D} q_{D}}{\mu_{S} q_{S}} M_{11}\right)}{\left(\frac{\mu_{D} q_{D}}{\mu_{A} q_{A}} M_{12}-\frac{\mu_{A} q_{A}}{\mu_{S} q_{S}} M_{21}\right)+i\left(M_{22}+\frac{\mu_{D} q_{D}}{\mu_{S} q_{S}} M_{11}\right)} .
\end{aligned}
$$

The energy transmittance $T(\omega)$ [reflectance $R(\omega)$ ] is defined as the ratio of the energy fluxes of incident and transmitted (reflected) phonons:

$$
\begin{gathered}
T(\omega) \equiv \frac{\mu_{D} q_{D}}{\mu_{S} q_{S}}|t(\omega)|^{2}, \\
R(\omega) \equiv|r(\omega)|^{2} .
\end{gathered}
$$

For simplicity, we consider the case in which $S=D=A$. In this case, Eq. (62) can be expressed in a simpler form

$$
T(\omega)=\frac{1}{1+\frac{1}{4} \varepsilon^{2} \sin ^{2} \alpha S^{2}(N)} .
$$

The effect of the multilayer structure is included in $S(N)$. Inside the frequency gap, it is found that

$$
T(\omega) \propto e^{-2 N P} \ll 1
$$

because $S(N) \propto e^{N P}$ [see Eq. (54)]. Inside the frequency band, on the other hand, $T(\omega)=1$ is realized. In particular, $T(\omega)$ becomes exactly unity for the frequencies satisfying $\sin (N P)=0$, i.e.,

$$
P=\frac{j}{N} \pi \quad(j=0,1,2, \ldots) .
$$

\section{NUMERICAL EXAMPLES AND DISCUSSION}

With the use of nanocluster catalysts, semiconductor NWSLs from group III-V and group IV materials have been synthesized. ${ }^{1-4}$ The radius of the NWSL is determined by the diameter of the nanocluster catalyst. On the other hand, the superlattice periodicity can be controlled by growth time. Typical dimensions have been nanowire radii of $10-50 \mathrm{~nm}$ and layer widths of 1-100 $\mathrm{nm}$.

As a numerical example, we present in Fig. 2 the phonon dispersion relations calculated for a NWSL consisting of the alternate stacking of GaAs and AlAs, which have been well studied in planar structures. ${ }^{11}$ In plain wires, there is no fundamental length scale within the continuum model. That is, the solution of the problem at one length scale (radius $R$ ) determines the solution at all other length scales. In NWSLs, on the other hand, the ratio between the radius $R$ and the periodicity $D$ becomes important. As the first example, we assumed that $R=5 D=42.5 \mathrm{~nm}$ in Fig. 2. As shown in Eq. (a) $s=0$

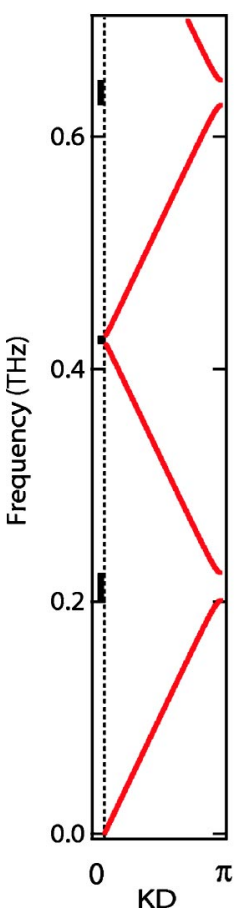

(b) $s=1$

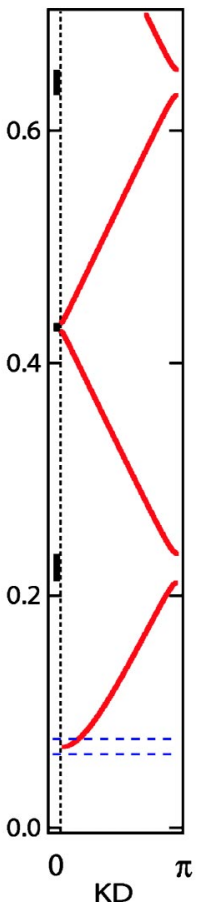

(c) $s=2$

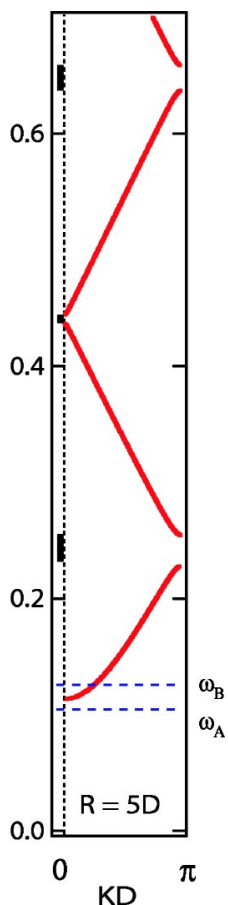

FIG. 2. The phonon dispersion relations of the (100) GaAs/AlAs NWSL with $R=5 D$. The unit period consists of $(\mathrm{GaAs})_{15}(\mathrm{AlAs})_{15}$. The parameters used are as follows: the thickness of one monolayer is $2.83 \AA$ in the (100) direction for both GaAs and AlAs; the mass densities and longitudinal sound velocities are $5.36 \mathrm{~g} / \mathrm{cm}^{3}$ and 4.71 $\mathrm{km} / \mathrm{s}$ for GaAs, and $3.76 \mathrm{~g} / \mathrm{cm}^{3}$ and $5.65 \mathrm{~km} / \mathrm{s}$ for AlAs. Solid vertical lines indicate frequency gaps determined by Eqs. (43), (47), and (48).

(11), the wave vector in the radial direction, $k_{t}$, is quantized due to the radial confinement. We show the results for the first three modes $(s=0,1,2)$. The overall structure of each phonon dispersion relation can be roughly understood by the folding of the dispersion curves for a homogeneous cylinder into a mini-Brillouin zone (BZ) determined by the periodicity $D$ of the NWSL. In this folded dispersion relation, the frequency gaps are generated at the center and edges of the mini-BZ due to Bragg reflections. As a result, the miniband structures are generated.

The frequency at the center of the $m$ th frequency gap and the width of the frequency gap are given in Eqs. (43) and (48), respectively. Equation (43) shows that the Bragg frequency due to the superlattice longitudinal confinement is determined by the radius $R$ of the NWSL. The frequency gaps determined by these approximate formulas are also shown in Fig. 2 (solid vertical lines). In the present example, the frequency gaps are well reproduced with the approximate formulas. These approximate formulas are valid when the condition (44) is satisfied, i.e., the effect of the radial confinement is regarded as a small perturbation. In the example illustrated in Fig. 2, this condition is satisfied. In a given structure of the NWSL (with fixed $R$ and $D$ ), the approximation becomes accurate for smaller $s$ and larger $m$ (i.e., higher frequency gap). For the NWSL with smaller $R$, the discrepancy becomes larger. As such an example, we show the dis- 
persion relations calculated for a NWSL with $R=D$. For $s$ $\geqslant 1$, the lowest frequency of each band is high compared with Fig. 2, because the wave length in the radial direction is smaller. The gap width depends on $s$ strongly in NWSLs with smaller $R$. This $s$ dependence is due to the higher order terms of $\left(f_{2, s} D / m R\right)^{2}$. Within the approximation we used, the gap width is independent of $s$, as shown in Eq. (48).

As mentioned before, the wave vector $q_{I}(I=A, B)$ defined within the layer $I$ is allowed to be an imaginary number.
When $\omega \geqslant v_{I} f_{2, s} / R \equiv \omega_{I}, q_{I}$ is a real number and the dependence of the displacement on $z$ has an oscillatory nature in the layer $I$ [see Eq. (19)]. When $\omega<\omega_{I}$, on the other hand, $q_{I}$ becomes an imaginary number and the displacement decays exponentially in the layer $I$. Thus, the displacement pattern is qualitatively different with the frequency range. The expression for the phonon dispersion relation (33) can be explicitly rewritten as

$$
\cos K D= \begin{cases}\cosh \alpha \cosh \beta+\frac{1}{2}\left(\frac{\mu_{B}\left|q_{B}\right|}{\mu_{A}\left|q_{A}\right|}+\frac{\mu_{A}\left|q_{A}\right|}{\mu_{B}\left|q_{B}\right|}\right) \sinh \alpha \sinh \beta & \text { for } \quad \omega \leqslant \omega_{A} \\ \cos \alpha \cosh \beta-\frac{1}{2}\left(\frac{\mu_{B}\left|q_{B}\right|}{\mu_{A}\left|q_{A}\right|}-\frac{\mu_{A}\left|q_{A}\right|}{\mu_{B}\left|q_{B}\right|}\right) \sin \alpha \sinh \beta & \text { for } \quad \omega_{A} \leqslant \omega \leqslant \omega_{B} \\ \cos \alpha \cos \beta-\frac{1}{2}\left(\frac{\mu_{B}\left|q_{B}\right|}{\mu_{A}\left|q_{A}\right|}+\frac{\mu_{A}\left|q_{A}\right|}{\mu_{B}\left|q_{B}\right|}\right) \sin \alpha \sin \beta & \text { for } \quad \omega_{B} \leqslant \omega,\end{cases}
$$

where $\alpha=\left|q_{A}\right| d_{A}, \beta=\left|q_{B}\right| d_{B}$. In Eq. (67), $v_{A}<v_{B}$ is assumed (i.e., $A=\mathrm{AlAs}$ and $B=\mathrm{GaAs}$ in the present example). The lines $\omega=\omega_{A}$ and $\omega=\omega_{B}$ are indicated in Fig. 3 (and also Fig. 2).

For $s=0$, there is no region determined by $\omega_{A} \leqslant \omega \leqslant \omega_{B}$ because $f_{2,0}=0$. Thus, the wave vector $q_{I}$ defined within each layer is always a real number. For $s=1$, the line $\omega=\omega_{B}$ inter- (a) $s=0$

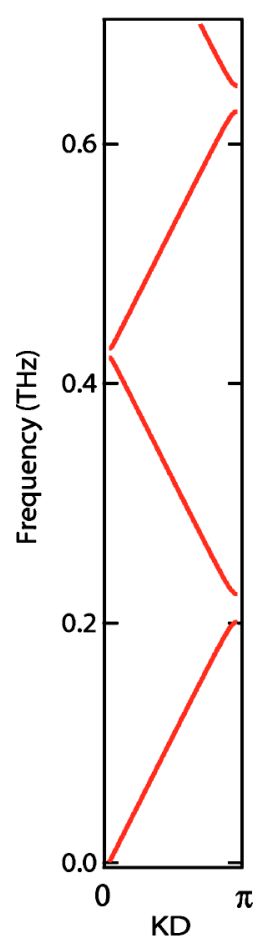

(b) $s=1$

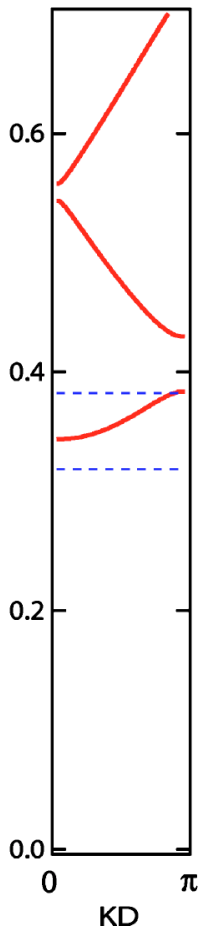

(c) $s=2$

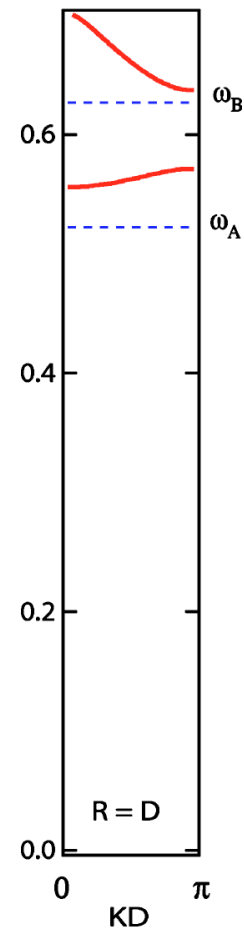

FIG. 3. The phonon dispersion relations of the (100) GaAs/AlAs NWSL. The radius is assumed to be $R=D$. sects the lowest frequency band. That is, in the lowest frequency band, the displacement pattern is different depending on whether the frequency is higher than $\omega_{B}$ or not. Below $\omega_{B}$, the wave vector $q_{B}$ becomes an imaginary number in this band. For $s=2$, the lowest frequency band is entirely located below $\omega_{B}$. Therefore, the wave vector in a layer with the lower sound velocity becomes an imaginary number in this band.

As we saw, there is a case where the wave vector defined in a layer becomes an imaginary number. However, the Bloch wave number $K$ defined by Eq. (33) is a real number in the frequency band. In the frequency gap, on the other hand, the Bloch wave number becomes an imaginary number. Such a vibrational mode is not allowed for the system with the infinite period. However, this mode may exist in NWSLs with the finite period. In Fig. 4, we show the transmittance calculated for a NWSL with $N=8$. We can see the large dips corresponding to the frequency gap. Transmittance has a nonvanishing value even in the frequency gap. This is due to the finite length in the longitudinal direction. The transmittance within the frequency gap is given by Eq. (65). In Eq. (65), $P$ means the decay factor of phonon displacement, which is defined by Eq. (56). In general, this decay factor has larger value in the wider frequency gap. Thus, the value of the transmittance becomes considerably small within the wide frequency gaps, such as the lowest gaps shown in Figs. 4(b) and 4(c).

\section{CONCLUDING REMARKS}

In the present paper, we studied the vibrational modes in a NWSL. The NWSL is modeled as an ideal cylinder with sharp modulation in the longitudinal direction. The eigenfrequencies of the vibrational modes in the NWSL are labeled with five quantum numbers: $p, s, m, K$, and $\ell$, where $p$ rep- 
(a) $s=0$

(b) $s=1$

(c) $s=2$
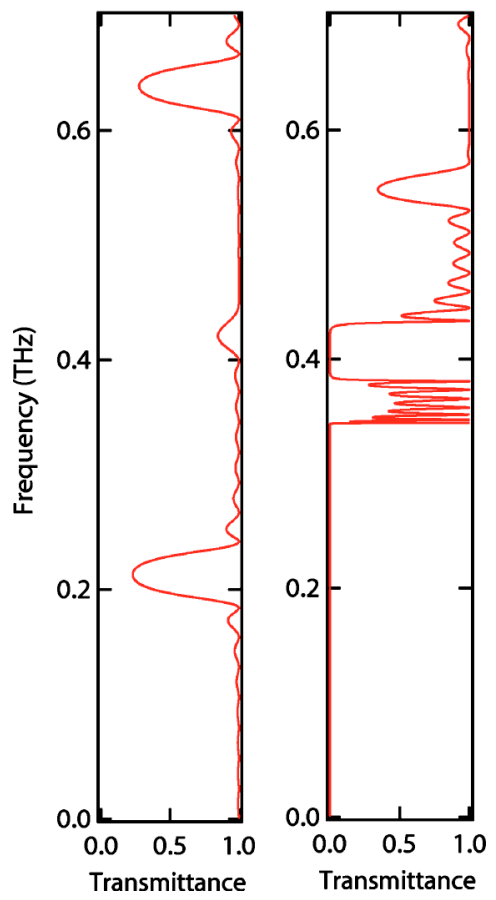

APPENDIX: DERIVATION OF EQ. (46)

The second term on the right-hand side of Eq. (33) has only a small contribution. Thus, Eq. (33) can be expanded around $\widetilde{\omega}_{m}$ as

$$
\begin{aligned}
\frac{m_{11}+m_{22}}{2} \cong & I\left(\widetilde{\omega}_{m}\right)+\left.\frac{1}{2}\left(\omega-\widetilde{\omega}_{m}\right)^{2} \frac{d^{2} I(\omega)}{d \omega^{2}}\right|_{\widetilde{\omega}_{m}} \\
& -\left.\frac{\varepsilon^{2}}{2} \sin \alpha \sin \beta\right|_{\widetilde{\omega}_{m}},
\end{aligned}
$$

where

$$
I(\omega)=\cos (\alpha+\beta) .
$$

From the definition of $\widetilde{\omega}_{m}$ [i.e., Eq. (39)], it is easily shown that

$$
I\left(\widetilde{\omega}_{m}\right)=(-1)^{m}
$$

and

$$
\left.\sin \alpha \sin \beta\right|_{\widetilde{\omega}_{m}}=(-1)^{m+1} \sin ^{2} \alpha_{m},
$$

where

$$
\alpha_{m}=\alpha\left(\widetilde{\omega}_{m}\right)=q_{A}\left(\widetilde{\omega}_{m}\right) d_{A} .
$$

At $\omega=\widetilde{\omega}_{m}, q_{A}$ and $q_{B}$ can be approximated as

$$
\begin{aligned}
& q_{A}\left(\widetilde{\omega}_{m}\right)=\frac{m \omega_{1}}{v_{A}}+\frac{f_{2, s}^{2}}{2 \pi m R^{2}}\left(\frac{v_{B}}{v_{A}}-\frac{v_{A}}{v_{B}}\right) d_{B}, \\
& q_{B}\left(\widetilde{\omega}_{m}\right)=\frac{m \omega_{1}}{v_{B}}+\frac{f_{2, s}^{2}}{2 \pi m R^{2}}\left(\frac{v_{A}}{v_{B}}-\frac{v_{B}}{v_{A}}\right) d_{A},
\end{aligned}
$$

respectively. By carrying out the straightforward calculation with the use of Eqs. (A6) and (A7), it is shown that

$$
\left.\frac{d^{2} I(\omega)}{d \omega^{2}}\right|_{\widetilde{\omega}_{m}} \cong(-1)^{m+1} \frac{\pi^{2}}{\omega_{1}^{2}}\left[1+\frac{f_{2, s}^{2}}{m^{2} \pi R^{2} \omega_{1}}\left(v_{A} d_{A}+v_{B} d_{B}\right)\right],
$$
coherent phonon generation or control, such as mirrors, filters, and also resonators. These are applicable to the micro/ nano electromechanical systems.

The results calculated analytically in the present paper are limited to the azimuthally symmetric torsional modes whose displacements are perpendicular to the $z$ axis. For these modes, it was possible to proceed the analytical calculation. The analysis of other modes can provide complementary information for fundamental understanding of the vibration generated in the NWSLs, though the analytical formulas cannot be obtained. The results calculated numerically for other modes will be given elsewhere.

\section{ACKNOWLEDGMENTS}

The author had useful discussions with N. Nishiguchi. He also acknowledges $\mathrm{S}$. Tamura for reading the manuscript and giving valuable advice.
Inserting Eqs. (A3), (A4), (A8), and (A9) into Eq. (A1) and neglecting small contribution terms, we have

$$
\frac{m_{11}+m_{22}}{2}=(-1)^{m}\left[1+\frac{\varepsilon_{0}^{2}}{2} \sin ^{2}\left(\omega_{m} d_{A} / v_{A}\right)-\frac{1}{2}\left(\omega-\widetilde{\omega}_{m}\right)^{2} \frac{\pi^{2}}{\omega_{1}^{2}}\right]
$$


${ }^{1}$ M. S. Gudikson, L. J. Lauhon, J. Wang, D. C. Smith, and C. M. Lieber, Nature (London) 415, 617 (2002).

${ }^{2}$ Y. Wu, R. Fan, and P. Yang, Nano Lett. 2, 83 (2002).

${ }^{3}$ M. T. Bjork, B. J. Ohlosson, T. Sass, A. I. Persson, C. Thelander, M. H. Magnusson, K. Deppert, L. R. Wallenberg, and L. Samuelson, Nano Lett. 2, 87 (2002).

${ }^{4}$ R. Solanki, J. Huo, J. L. Freeouf, and B. Miner, Appl. Phys. Lett. 81, 3864 (2002).

${ }^{5}$ L. C. Lew Yan Voon and M. Willatzen, J. Appl. Phys. 93, 9997 (2003).
${ }^{6}$ A. N. Cleland and M. L. Roukes, Nature (London) 392, 160 (1998).

${ }^{7}$ V. Narayanamurti, Science 213, 717 (1981).

${ }^{8}$ V. G. Grigoryan and D. G. Sedrakyan, Sov. Phys. Acoust. 29, 281 (1983).

${ }^{9}$ B. A. Auld, in Acoustic Fields and Waves in Solids (Krieger, Malabar, 1990), Vol. 2.

${ }^{10}$ N. Nishiguchi, Phys. Rev. B 50, 10970 (1994).

${ }^{11} \mathrm{~S}$. Mizuno, in Recent Reserch Developments in Physics (Transworld Research Network, Kerala, 2002), Vol. 3, p. 379. 